placebo-controlled trial / C. Baigent, M. Landray, C. Reith [et al.] // Lancet. - 2011. - Vol. 377. - P.2181-2192.

11. Мельник, А.А. Нарушение липидного обмена и его коррекция при хронической болезни почек / А.А. Мельник // Почки. - 2016. - № 2 (16). - С.85-95.

12. Меншутина, М.А. Эндотелиальная дисфункция у больных хронической болезнью почек / М.А. Меншутина, И.Ю. Панина, А.В. Смирнов // Нефрология. - 2004. № 3 (2). - C.48-54.

13. Колина, И.Б. Гиперлипидемии при хронической болезни почек: особенности и подходы к лечению / И.Б. Колина // Лечащий врач. - 2012. - № 1. - С.23-25.

14. The vasodilatator action of nebivalol in forearm vasculature of subjects with essential hypertension / M. Daweas, S. Brett, P. Chowienczyk [et al.] // Br. J. Clin. Pharmacol. 1994. -Vol. 148. - P.460-463.

\section{REFERENCES}

1. Romanovskaya GA, Akatova EV, Gorohovskaya GN Perspektivy medikamentoznogo lecheniya endotelial'noj disfunkcii. [Prospects for medical treatment of endothelial dysfunction]. Farmateka [Pharmateca]. 2005; 9: 31-37.

2. Vlasova SP, Il'chenko MYu, Kazakova EB. Endotelial'naya disfunkciya i arterial'naya gipertenziya [Endothelial dysfunction and hypertension]. Samara: OOO «Ofort». 2010: 192 p.

3. Galyavich AS. Sovremennaya farmakoterapiya pacientov s arterial'noj gipertenziej i vysokim riskom serdechnososudistyh oslozhnenij: $v$ fokuse ingibitory angiotenzinprevrashchayushchego fermenta [Modern pharmacotherapy of patients with arterial hypertension and high risk of cardiovascular complications: angiotensin-converting enzyme inhibitors are in focus]. Medicinskij sovet [ Medical Council]. 2012; 12: 10-17.

4. Demin AA, Shulyat'eva OYu. Novye vozmozhnosti blokatora receptov angiotenzina II telmisartana $v$ lechenii arterial'noj gipertenzii s metabolicheskimi narusheniyami [New features of the angiotensin II prescription blocker telmisartan in the treatment of hypertension with metabolic disorders]. Klinicheskaya medicina [Clinical medicine]. 2012; 1: 32-34.

5. Martynov Al, Gorohovskaya GN. Perspektivy medikamentoznogo lecheniya endotelial'noj disfunkcii [Prospects for the medical treatment of endothelial dysfunction]. Farmateka [Pharmateca]. 2005; 9: 31-40.

6. Tan K, Chow W, Ai V. Effects of angiotensin II reseptor antagonists on endothelial vasomotor function and urinary albumin excretion in type 2diabetic patients with microalbuminuria. Diabetic Metabolism Research and Reviews. 2002; 18 (1): 71-76.

7. Markova LI, Radzevich AE, Lazarev AV. Optimizaciya kombinirovannoj terapii pri trudnokontroliremoj arterial'noj gipertenzii [Optimization of combination therapy for difficult-to-control arterial hypertension]. Zhurnal Lechashchij vrach [Journal Physician]. 2012; 7: 4-8.

8. Fedoseev AN, Kudryakova AS, Smirnov VV. Primenenie ingibitorov APF s cel'yu korrekcii endotelial'noj disfunkcii u pacientov na raznoj stadii hronicheskoj bolezni pochek [The use of ACE inhibitors to correct endothelial dysfunction in patients at different stages of chronic kidney disease]. Klinicheskaya nefrologiya [Clinical Nephrology]. 2012; 4: 10-15.

9. Nebieridze DV. Disfunkciya endoteliya i ee korrekciya pri arterial'noj gipertonii [Endothelial dysfunction and its correction in arterial hypertension]. Russkij medicinskij zhurnal [Russian Medical Journal]. 2006; 14 (2): 34-38.

10. Baigent $C$, Landray M, Reith $C$ et al. The effects of lowering LDL cholesterol with simvastatin plus ezetimibe in patients with chronic kidney disease (Study of Heart and Renal Protection): a randomised placebo-controlled trial. Lancet. 2011; 377: 2181-2192.

11. Mel'nik AA. Narushenie lipidnogo obmena i ego korrekciya pri hronicheskoj bolezni pochek [Disruption of lipid metabolism and its correction in chronic kidney disease]. Pochki [Kidneys]. 2016; 2 (16): 85-95.

12. Menshutina MA, Panina IYu, Smirnov AV. Endotelial'naya disfunkciya u bol'nyh hronicheskoj bolezn'yu pochek [Endothelial dysfunction in patients with chronic kidney disease]. Nefrologiya [Nephrology]. 2004; 3 (2): 48-54.

13. Kolina IB. Giperlipidemii pri hronicheskoj bolezni pochek: osobennosti i podhody $\mathrm{k}$ lecheniyu [Hyperlipidemia in chronic kidney disease: features and approaches to treatment]. Lechashchij vrach [Physician]. 2012; 1: 23-25.

14. Daweas M, Brett S, Chowienczyk P et al. The vasodilatator action of nebivalol in forearm vasculature of subjects with essential hypertension. Br J Clin Pharmacol. 1994; 148: 460-463.

\title{
ПРОБЛЕМЫ ДИАГНОСТИКИ
}

\section{И ФАКТОРЫ ФОРМИРОВАНИЯ НАРУШЕНИЙ СЛУХА У ЧЛЕНОВ ЛЕТНЫХ ЭКИПАЖЕЙ ГРАЖДАНСКОЙ АВИАЦИИ}

АДЕНИНСКАЯ ЕЛЕНА ЕВГЕНЬЕВНA, ORCID ID: 0000-0001-7483-3855; канд. меД. наук, руководитель научно-исследовательского центра профпатологии и гигиены труда гражданской авиации ФБУ «Центральная клиническая больница гражданской авиации», Россия, 125367, Москва, Иваньковское шоссе, 7,

e-mail: Ioruna@gmail.com

СИМОНОВА НАДЕЖДА ИВАНОВНА, докт. мед. наук, профессор, директор департамента по науке ЗАО «Клинский институт охраны и условий труда», Россия, 141607, Клин, Московская обл.,

ул. Дзержинского, 6, e-mail: simonovani@yandex.ru

МАЧАЛОВ АНTOH CEPTEEBИЧ, ORCID ID: 0000-0002-5706-7893; канд. меД. наук, начальник научно-клинического отдела аудиологии, слухопротезирования и слухоречевой реабилитации ФГБУ НКЦ оториноларингологии ФМБА России, Россия, 123182, Москва, Волоколамское шоссе, 30, стр. 2, e-mail: anton-machalov@mail.ru

\footnotetext{
Реферат. Цель исследования - научное обоснование алгоритмов диагностики и экспертизы нарушений слуха у членов летных экипажей гражданской авиации с учетом фракторов их формирования. Материал и методы. Проведено проспективное исследование в ретроспективно ссоорированной когорте с использованием современных аудиологических, клинических, социально-гигиенических и статистических методов исследования. Объектом исследования являлось состояние слуховой функции и здоровья членов летных экипажей граждан-
} 
ской авиации - мужчин ( $n=2224$ чел.). Результаты и их обсуждение. Показано, что у $70 \%$ обследованных летчиков фрактические пороги слышимости не соответствуют клинической форме тугоухости и укладываются в диапазон нормальных значений. Нарушения слуха у остальных летчиков в большинстве случаев не выходят за границы параметров первой степени тугоухости. Установлено, что авиационный шум вызывает повышение порогов слышимости на частотах 3 и 4 кГц, причем зависимость возникает при уровне шума не ниже 80 дБА и увеличивается с его нарастанием. Пороги слышимости на частотах 3 и 4 кГц не зависят от длительности полетного времени, а на частотах 1, 2, 6 и 8 кГц - от авиационного шума. Выводы. Доказана перспективность использования объективных методов оценки состояния слуха работников в трудоспособном возрасте и обоснованы критерии их применения.

Ключевые слова: шумовой пороговый сдвиг, возрастная потеря слуха, профессиональная потеря слуха.

Для ссылки: Аденинская, Е.Е. Проблемы диагностики и фракторы фрормирования нарушений слуха у членов летных экипажей гражданской авиации / Е.Е. Аденинская, Н.И. Симонова, А.С. Мачалов // Вестник современной клинической медицины. - 2019. - Т. 12, вып. 6. - С.12-18. DOI: 10.20969/VSKM.2019.12(6).12-18.

\title{
PROBLEMS OF DIAGNOSIS AND THE FACTORS OF HEARING DISORDERS IN CIVIL AVIATION FLIGHT CREW MEMBERS
}

\author{
ADENINSKAYA ELENA E., ORCID ID: 0000-0001-7483-3855; C. Med. Sci., Head of the research center \\ of occupational medicine and industrial hygiene of Central Clinical Hospital of Civil Aviation, Russia, 125367, \\ Moscow, Ivankovsk highway, 7, e-mail: loruna@gmail.com \\ SIMONOVA NADEZHDA I., D. Med. Sci., professor, Director of the Department for science of Klin Institute \\ of Occupational Safety and Working Conditions, Russia, 141607, Klin, Moscow region, Dzerzhinsky str., 6 , \\ e-mail: simonovani@yandex.ru \\ MACHALOV ANTON S., ORCID ID: 0000-0002-5706-7893; C. Med. Sci., Head of the Department of clinical \\ research audiology, hearing and hearing rehabilitation of Research and Clinical Center of Otorhinolaryngology \\ FMBA of Russia, Russia, 123182, Moscow, Volokolamsk highway, 30, build. 2, e-mail: anton-machalov@mail.ru
}

\begin{abstract}
The purpose of the study is the scientific substantiation of algorithms for diagnosis and examination of hearing impairment in members of flight crews of civil aviation, considering the factors of their formation. Material and methods. A prospective study was conducted in a retrospectively formed cohort using modern audiological, clinical, socio-hygienic and statistical methods. The object of the study was the state of auditory function and health of members of flight crews of civil aviation - men ( $n=2224$ people). Results and its discussion. It is shown that in $70 \%$ of the examined pilots the actual hearing thresholds do not correspond to the clinical form of hearing loss and fit into the range of normal values. Hearing impairment in other pilots in most cases do not go beyond the parameters of the first degree of hearing loss. It is established that aircraft noise causes an increase in the hearing thresholds at frequencies of 3 and $4 \mathrm{kHz}$, and the dependence occurs at a noise level of not less than $80 \mathrm{dBA}$ and increases with its increase. Hearing thresholds at frequencies 3 and $4 \mathrm{kHz}$ do not depend on the duration of the flight time, and at frequencies 1, 2, 6 and $8 \mathrm{kHz}$ - on aircraft noise. Conclusion. The prospects of the use of objective methods for assessing the state of hearing of workers at working age are proved and the criteria for their application are substantiated.

Key words: noise-induced permanent threshold shifts, age-related hearing loss, noise induced hearing loss.

For reference: Adeninskaya EE, Simonova NI, Machalov AS. Problems of diagnosis and the factors of hearing disorders in civil aviation flight crew members. The Bulletin of Contemporary Clinical Medicine. 2019; 12 (6): 12-18. DOI: 10.20969/ VSKM.2019.12(6).12-18.
\end{abstract}

B ведение. К настоящему времени в Российской Федерации (РФ) сформировалось выраженное противоречие с экономически развитыми странами в отношении частоты выявления потери слуха, вызванной шумом (ПСВШ), у членов летных экипажей гражданской авиации (ГА), причем на фроне отсутствия корреляции между фрактическими уровнями шума в кабинах воздушных судов (BC) и показателями заболеваемости [1, 2]. В последние годы фрормируется устойчивая практика признания нарушения слуха одной из основных причин негодности к выполнению летной работы членов летных экипажей в РФ, а их удельный вес в структуре причин негодности достигает 85\%. При этом в абсолютном большинстве случаев, независимо от исходного клинического диагноза, нарушения слуха у пилотов связывают с шумом, воздействию которого они подвергаются в полете: у них ежегодно устанавливают от 500 до 600 случаев профессиональной тугоухости. Важнейшей, на наш взгляд,

особенностью сложившейся ситуации является широкое вовлечение в нее специалистов из области медицины труда и профессиональной патологии и практически полное отсутствие репрезентативных исследований, касающихся вопросов клинико-аудиологического обследования членов летных экипажей, доказательств наличия аудиологических признаков воздействия шума, выявления основных причин нарушений слуха на низких и высоких частотах и проведения соответствующей дифференциальной диагностики [2, 3].

Все это не может не вызывать обоснованных сомнений в качестве диагностики по следующим причинам: в мировой практике отсутствует проблема нарушения слуховой функции и его связи с профессией у членов летных экипажей [4]; отсутствует корреляция между условиями труда и выявлением профессиональной тугоухости [5]; основным, а зачастую единственным методом диагностики нарушений слуха у работников, за- 
нятых в условиях воздействия шума, традиционно является тональная пороговая аудиометрия (ТПА), тогда как объективные аудиологические методы исследования практически не используются. Кроме того, при диагностике ПСВШ не учитываются фризиологические возрастные изменения слуха, что особенно актуально для старших возрастных групп. До настоящего время не согласованы требования к допуску летного состава с нарушением слуховой функции к летной работе и к работе в условиях шума вследствие противоречий в действующих нормативных документах [6].

Перечисленные выше обстоятельства определили цель настоящего исследования - научное обоснование алгоритмов диагностики и экспертизы нарушений слуха у членов летных экипажей гражданской авиации с учетом фракторов их формирования.

Материал и методы. Основным объектом исследования в настоящей работе является состояние слуховой функции и здоровья авиационных специалистов-мужчин, являющихся членами летных экипажей гражданской авиации ( $n=2224$ чел.). Выполненное исследование является проспективным в ретроспективно сформированной когорте с преимущественным использованием принципа сплошной выборки. В работе использованы современные аудиологические, клинические, социальногигиенические и статистические методы исследования. Оценка степени тугоухости проводилась в соответствии с Международной классификацией степени нарушения слуха, гармонизированной с учетом требований охраны труда и безопасности на рабочем месте [6]. Для анализа полученных материалов использованы стандартные методы статистической обработки, включая вариационную и непараметрическую статистику, корреляционный, дисперсионный, факторный анализ, метод множественной регрессии с построением логистических моделей регрессионного анализа, а также кластерный анализ. Статистически значимое различие определялось при $p<0,05$.

Результаты и их обсуждение. В наблюдаемой когорте членов летных экипажей ( $n=2224$ чел.) средний профессиональный стаж составил [M (95\% дИ)] 32,8 (32,5-33,1) года; возраст [M (95\% ДИ)] -
$56,6(56,3-56,8)$ года; налет [М (95\% ДИ)] - 12734,2 $(1253,7-12954,6)$ ч. В $70 \%$ случаев стаж летной работы превышал 30 лет, причем 78,3\% пилотов имели возраст старше 55 лет, около 30\% - старше 60 лет [7].

У 1246 (56\%) человек ранее на врачебно-летной экспертной комиссии (ВЛЭК) был установлен диагноз «двусторонняя сенсоневральная тугоухость» (ДСНТ). Средняя длительность наблюдения на ВЛЭК с установленным диагнозом ДСНТ составила 8,4 (5,3-11,6) года, средний стаж работы на период первой диагностики тугоухости - 26,5 (23,3-29,7) года. Последующий детальный анализ аудиологических характеристик слуха у летного состава проводился на основании исследований, проведенных в рамках настоящего исследования.

По результатам проведенной ТПА установлено, что регистрируемое в стране большое количество вновь выявляемых случаев ПСВШ у членов летных экипажей не соответствует фактической картине состояния слуха у обследованных работников. В действительности почти в $70 \%$ случаев фрактические пороги слуха укладываются в нормальные значения порогов слышимости (табл. 1) [7].

Лишь у $26 \%$ обследованных выявлена двусторонняя тугоухость, причем только у $2 \%$ - II степени. Еще у 4\% обследованных нарушения слуха характеризуются выраженной асимметрией с разницей порогов слышимости правового и левого уха не менее 15 дБ [7].

Согласно результатам проведенного ретроспективного анализа между данными архивных ТПА и исследованиями, выполненными в рамках настоящей работы, выявлено большое количество расхождений как в оценке фактического состояния слуха, так и в степени его потери. В 40\% случаев фракт выявленной ранее тугоухости не был подтвержден (рис. 1).

Помимо этого, следует иметь в виду, что среди лиц с тугоухостью $82 \%$ обследованных были в возрасте старше 55 лет, т.е. их возрастные пороги слышимости существенно выше параметров условной нормы, установленной для 18-летних. Очевидно, что в ряде случаев они могли ошибочно трактоваться как тугоухость, в том числе вызванная шумом $[2,7]$.

Аудиологическая характеристика порогов слышимости у членов летных экипажей

\begin{tabular}{|l|c|c|c|}
\hline \multirow{2}{*}{ Оценка состояния слуха } & \multirow{2}{*}{$\begin{array}{c}\text { Число } \\
\text { обследованных, } n\end{array}$} & \multicolumn{2}{|c|}{$\begin{array}{c}\text { Средний порог слышимости (М) на частотах } \\
0,5,1,2 \text { и } 4 \text { кГц, дБ }\end{array}$} \\
\cline { 3 - 4 } & & $\mathrm{AD}$ & $\mathrm{AS}$ \\
\hline Условная норма - 0-25 дБ & $986(69,4 \%)^{1}$ & $15,4(15,0-15,8)^{2}$ & $3,1(14,7-15,5)^{2}$ \\
\hline $\begin{array}{l}\text { Двусторонняя тугоххсть І степени - } \\
\text { 26-40 дБ }\end{array}$ & $341(24,0 \%)$ & $29,3(28,7-30,0)$ & $31,2(30,8-31,6)$ \\
\hline $\begin{array}{l}\text { Двусторонняя тугоухость II степени - } \\
\text { 41-55 дБ }\end{array}$ & $32(2,3 \%)$ & $40,6(38,5-42,6)^{*}$ & $44,5(43,0-46,0)^{*}$ \\
\hline Тугоухость с асимметрией $\geq 15$ дБ & $61(4,3 \%)$ & $26,8(23,0-30,5)$ & $33,8(29,9-37,7)$ \\
\hline Итого & $1420(100,0 \%)$ & $28,0(15,0-30,5)$ & $31,2(14,7-37,7)$ \\
\hline
\end{tabular}

Примечание: в скобках указан: ${ }^{1} \%$ от общей численности лиц в выборке $(n=1420) ;{ }^{2}$ доверительный интервал (ДИ); ${ }^{*}$ различия статистически достоверны, $p<0,01$. 


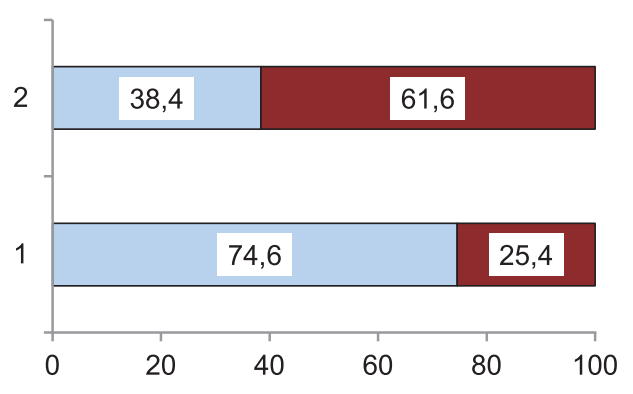

$\square$ Норма Нарушение порогов слышимости

Рис. 1. Заключение ТПА в период обследования: 1 - в рамках проводимого исследования; 2 - на ВЛЭК

Речевая аудиометрия, проведенная в различных группах обследованных, показала, что у пилотов длительно сохраняется разборчивость речи, которая может не достигать 100\%, как правило, при II степени тугоухости, однако достоверность исследования уровня разборчивости речи четко зависит от мотивации работника на летную работу. Полученные результаты надпороговой аудиометрии свидетельствуют о том, что между величиной значений $\mathrm{SiSi-теста} \mathrm{и} \mathrm{уровнем}$ порогов слышимости существует довольно строгая корреляция. Работники, которые имели признаки ложных ответов при ТПА, то же самое демонстрируют и при прохождении SiSi-теста. Проведенный анализ в очередной раз подтвердил субъективность данных методов исследования и необходимость расширения обследования работников при помощи объективных методов исследования органа слуха.

По результатам тимпанометрии практически у всего летного состава зарегистрирована тимпанограмма типа «А», а пороги акустической рефлексометрии при ипсилатеральной (+ контра) стимуляции соответствуют нормальным значениям. Для показателей отоакустической эмиссии (ОАЭ) определена зависимость получения ответа от шума, возраста, стажа, налета и сопутствующей соматической патологии. Установлено, что на величину соотношения «сигналшум» практически на всех анализируемых частотах с большой вероятностью влияет возраст работника и длительность полетного времени. Получена слабая зависимость на частоте 2 кГц от наличия сердечно- сосудистой патологии (ССП), установлено отсутствие зависимости от стажа и уровня воздействия шума. Выявлено наличие зависимости между соотношением «сигнал-шум» и порогом слышимости как при задержанной вызванной отоакустической эмиссии, так и на частоте продукта искажения (рис. 2) [7].

Подтверждено, что получение удовлетворительных результатов ОАЭ при наличии признаков значимой тугоухости на ТПА, как правило, свидетельствует о недостоверности либо искажении результатов аудиометрии. Это подтверждает обоснованность использования ОАЭ при оценке состояния слуха в случае противоречивых результатов ТПА.

Для получения количественных соотношений между порогами слышимости и показателями ОАЭ проведен кластерный анализ. Полученные результаты четко подтверждают взаимосвязь прохождения эмиссии с результатами аудиометрии на популяционном уровне. Поскольку получена статистически значимая зависимость показателя «сигнал-шум» от возраста, следует иметь в виду, что непрохождение теста может иметь место и при отсутствии аудиометрических признаков нарушения слуха, особенно у лиц старших возрастных групп. Это является одним из основных показаний для проведения ASSR-теста и регистрации коротколатентных вызванных потенциалов (КСВП).

У обследованных работников пики КСВП четко идентифицируются, что дало основание исключить наличие центрального поражения слухового анализатора. Полученные результаты свидетельствуют о наличии у обследованных работников фактических порогов слышимости с нормальными значениями в пределах статистической погрешности. Порог визуализации V-пика на КСВП совпадал с тональным порогом слышимости на частотах 2-3 кГц и находился в пределах статистической погрешности на частоте 4 кГц. Усредненные почастотные показатели порогов слышимости при TПА и ASSR-тесте полностью совпадают на частотах речевого диапазона $(0,5,1$ и 2 кГц). Основные расхождения отмечены на частоте 4 кГц, когда пороги ASSR в среднем на 12 дБ ниже, чем при ТПА. Несовпадение данных было характерно для пациентов с заведомо ложными порогами слышимости на ТПА, что подтверждает информативность метода и позволяет рекомендовать

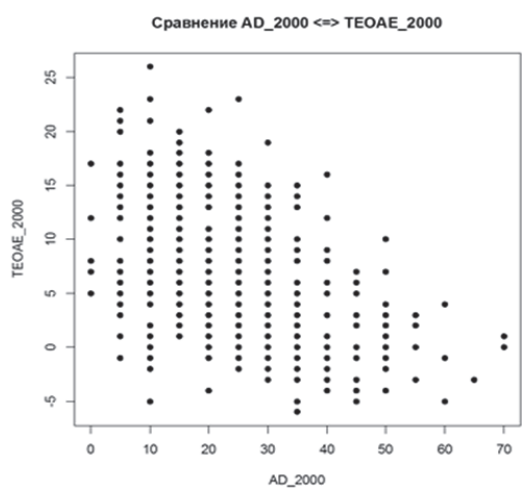

$\mathbf{A}$
$p=2 \mathrm{e}^{-16 * * *}$
$\mathrm{R}^{2}=0,1574$

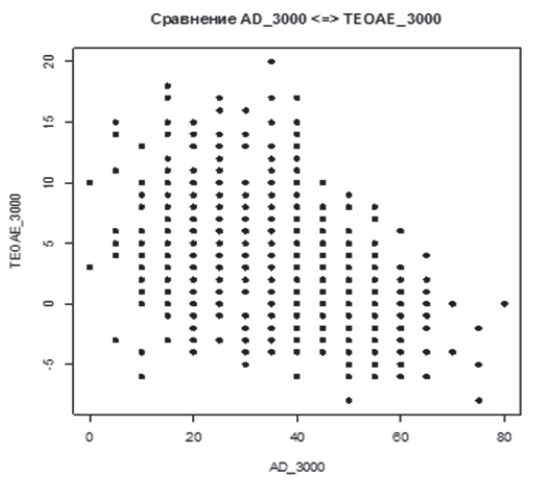

Б $p=2 \mathrm{e}^{-16 * * *}$ $\mathrm{R}^{2}=0,1737$

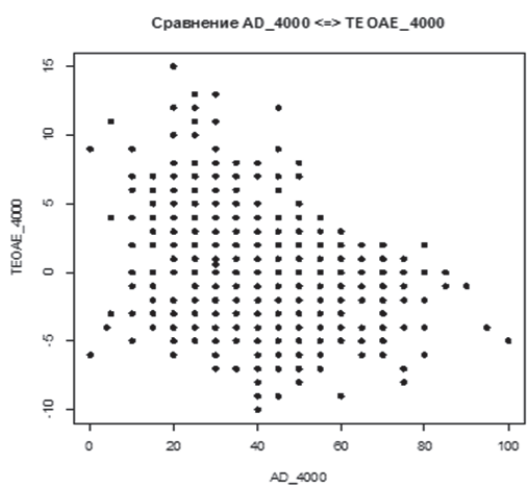

B $p=2 \mathrm{e}^{-16 * * *}$ $\mathrm{R}^{2}=0,09845$

Рис. 2. Зависимость между ОАЭ (ТЕОАЕ) и порогом слышимости ТПА на частотах 2 (А), 3 (Б) и 4 (В) кГц 
его в качестве метода выбора при необходимости решения сложных экспертных вопросов, связанных с анализом противоречивых результатов ТПА.

Для обоснования алгоритма принятия решения о годности к летной работе проведен анализ тональных порогов слышимости пилотов, признанных негодными по состоянию органа слуха, в результате чего выявлено, что в $90 \%$ случаев пороги слышимости у них не превышают предельных значений требований федеральных авиационных правил (ФАП МО ГА-2002).

Установлено, что у признанных негодными к летной работе членов летных экипажей в группе обследования средний возраст составил 58,2 (95\% ДИ: 57,2$59,3)$ года, средний стаж работы - 34,2 $(33,0-35,5)$ года, общее полетное время - 13998,1 (13014,3-14981) ч. При этом шум в кабине ВС в среднем составлял 81,1 (79,7-82,6) дБА при норме 80 дБА, а длительность наблюдения на ВЛЭК с установленным диагнозом ДСНТ - 8,2 (7,0-9,4) года (табл. 2) [7].

Кроме того, установлено, что пороги слышимости на частоте 3 кГц, как правило, существенно зависящие от шума, статистически достоверно не отличаются у пилотов, признанных негодными к летной работе, и у тех, кто продолжает летать. Более того, признание члена летного экипажа негодным к летной работе существенно не зависело даже от степени тугоухости. Следовательно, существующая практика признания члена летного экипажа негодным к летной работе по состоянию слуха фактически никак не связана с реальными порогами слышимости на частоте 3 кГц.

Установлено, что понятие «визуальный анализ профиля аудиометрической кривой» имеет относительно невысокую диагностическую значимость, не является определяющим как для идентификации степени потери слуха, так и для оценки этиологи- ческих фракторов, его обусловивших, и может быть интерпретировано не более как основание для формирования исходных гипотез.

Поскольку в настоящее время тугоухость диагностируется без коррекции по возрасту, нами были проведены расчеты с целью получения статистического распределения порогов слышимости в возрасте от 20 до 75 лет с шагом в один год на основе ГОСТ Р ИСО 7029-2011 с вероятностью 0,5, 0,25 и 0,1. Для удобства анализа и оценки наличия признаков возрастной нормы слуха в практике оториноларинголога подготовлены соответствующие вспомогательные таблицы. Разработан алгоритм учета возрастной фризиологической нормы слуха, где за минимальное значение вероятности принята медиана, ниже которой эффект рассматривается как несущественный. Разработан алгоритм выявления и оценки шумового порогового сдвига (ШПС), величина которого должна быть не менее чем на 10 дБ выше возрастного порога слуха на частотах 3 и 4 кГц для принятия гипотезы о наличии у работника признаков шумовой потери слуха и обоснования объема последующих обследований с целью оценки ее выраженности.

Для выявления факторов, способных влиять на ухудшение слуха, проведен дисперсионный анализ зависимости потери слуха, в котором независимыми переменными были шум, стаж, полетное время и наличие болезней системы кровообращения.

Установлено, что внутрикабинный шум вызывает у членов летных экипажей повышение порогов слышимости на частотах 3 и 4 кГц, причем начальные признаки зависимости возникают не ранее чем при уровне шума 80 дБ. Повышение порогов слышимости на частотах 1, 2, 6 и 8 кГц не зависит от влияния внутрикабинного шума (рис. 3) [7].

Та блица 2

Аудиологическая характеристика усредненных порогов слышимости у членов летных экипажей, признанных в 2015-2017 гг. негодными к летной работе, $n=114$

\begin{tabular}{|c|c|c|c|c|c|c|c|}
\hline $\begin{array}{c}\text { Частота, } \\
\text { кГи }\end{array}$ & \multicolumn{5}{|c|}{ Усредненные результаты тональной пороговой аудиометрии, М (95\% ДИ), дБ } \\
\cline { 2 - 8 } & 0,5 & 1 & 2 & 3 & 4 & 6 & 5 \\
\hline AD & 21,7 & 24,7 & 31,5 & 42,8 & 52,1 & 50,8 & 52,3 \\
& $(19,8-23,6)$ & $(23,0-26,4)$ & $(29,2-33,9)$ & $(40,3-45,2)$ & $(49,7-54,6)$ & $(48,0-53,7)$ & $(49,0-55,6)$ \\
\hline AS & 23,4 & 26,1 & 33,8 & 45,8 & 53,7 & 53,2 & 54,8 \\
& $(21,5-25,3)$ & $(24,0-28,2)$ & $(31,4-36,2)$ & $(43,4-48,1)$ & $(51,3-56,2)$ & $(50,3-56,1)$ & $(51,7-57,9)$ \\
\hline
\end{tabular}
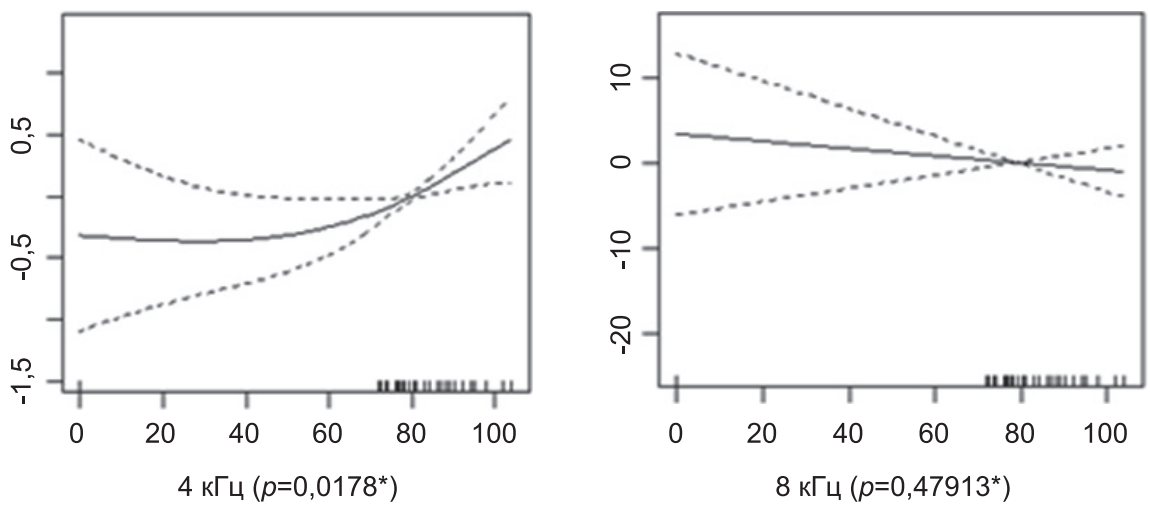

Рис. 3. Модели логистической регрессии: частные зависимости порогов слышимости от уровня внутрикабинного авиационного шума 
Наиболее высокий уровень зависимости порогов слышимости от стажа работы выявлен на частотах 4, 6 и 8 кГц. При этом с увеличением стажа работы свыше 20 лет степень зависимости резко снижается. Установлено, что повышение порогов слышимости не зависит от длительности полетного времени в области восприятия на частотах 3, 4 и 6 кГц. В речевом диапазоне частот 0,5, 1 и 2 кГц выявлена сильная статистическая значимость повышения порогов слышимости от полетного времени.

В исследуемой когорте лишь у 27,7\% лиц не было сопутствующих болезней системы кровообращения. Более чем у половины пилотов к началу проведения исследования был диагностирован атеросклероз аорты и сосудов головного мозга, у каждого четвертого имело место его сочетание с гипертонической болезнью (ГБ) 1-3-й степени, ИБС, либо сахарным диабетом (СД). Выявлена сильная зависимость повышения порогов слышимости у летного состава на всех частотах от наличия атеросклероза аорты и сосудов головного мозга, а также ГБ. При более выраженных и осложненных формах патологии системы кровообращения выявлена статистически значимая зависимость ухудшения слуха в речевом диапазоне (табл. 3).

Проведенные клинико-экспертные исследования позволили обосновать ряд алгоритмов аудиологического обследования членов летных экипажей [7].

\section{Выводы:}

1. Полученные данные о состоянии слуха у членов летных экипажей не подтверждают широкую распространенность у них шумовой патологии. У $70 \%$ обследованных летчиков фрактические пороги слышимости не соответствуют клинической форме тугоухости и укладываются в диапазон нормальных значений. Нарушения слуха у остальных летчиков в большинстве случаев не выходят за границы параметров 1-й степени тугоухости.

2. Для диагностики нарушений слуха у членов летных экипажей гражданской авиации необходимо использовать все основные аудиологические методы исследования, включая объективные, что определяется задачами оценки пригодности к летной работе, дифференциальной диагностики, экспертизы связи заболевания с профессией, а также высокой вероятностью низкого качества аудиоархива.

3. Члены летных экипажей с нарушением слуха могут быть признаны негодными к летной работе преимущественно при повышении порогов слышимости на речевых частотах, что не связано с воздействием внутрикабинного шума. При повышении порогов слышимости на частотах 3 и 4 кГц у членов летных экипажей длительно сохраняется разборчивость речи, что, как правило, позволяет обосновать индивидуальное экспертное решение о годности к летной деятельности.

4. В процессе дифференциальной диагностики потери слуха, вызванной шумом, необходимо учитывать медианную возрастную физиологическую норму слуха, ниже которой эффект рассматривается как несущественный.

5. Авиационный шум вызывает у членов летных экипажей повышение порогов слышимости на частотах 3 и 4 кГц, причем зависимость возникает при уровне шума 80 дБА и увеличивается с его нарастанием. Пороги слышимости на частотах 3 и 4 кГц не зависят от длительности полетного времени, а на частотах 1, 2, 6 и 8 кГц - от авиационного шума. Наличие сопутствующей патологии системы кровообращения способствует формированию тугоухости на всех частотах конвенционального диапазона.

6. Для принятия гипотезы о наличии у работника признаков шумовой потери слуха необходима оценка шумового порогового сдвига как разности между фактическим порогом слышимости и медианным значением возрастной физиологической нормы. Величина шумового порогового сдвига должна не менее чем на 10 дБ быть выше возрастного порога слуха на частотах 3 и 4 кГц.

Прозрачность исследования. Исследование не имело спонсорской поддержки. Авторы несут полную ответственность за предоставление окончательной версии рукописи в печать.

Декларация о финансовых и других взаимоотношениях. Все авторы принимали участие в разработке концепции, дизайна исследования и в написании рукописи. Окончательная версия рукописи была одобрена всеми авторами. Авторы не получали гонорар за исследование.

Оценка зависимости формирования потери слуха от наличия сопутствующей сердечно-сосудистой патологии

\begin{tabular}{|c|c|c|c|c|}
\hline $\begin{array}{c}\text { Пороги слышимости } \\
\text { на частотах, кГи }\end{array}$ & $\begin{array}{c}\text { Aтеросклероз сосудов } \\
(52,5 \%)\end{array}$ & $\begin{array}{c}\text { ГБ 1-й степени } \\
(10,1 \%)\end{array}$ & $\begin{array}{c}\text { ГБ 2-3-й степени } \\
(13,8 \%)\end{array}$ & $\begin{array}{c}\text { Сочетание ГБ с ИБС, } \\
\text { СД и др. }(1,9 \%)\end{array}$ \\
\hline 0,5 & $1,7 \mathrm{e}^{-05 * *}$ & 0,658 & $0,00729^{* *}$ & $0,00205^{* *}$ \\
\hline 1 & $1,44 \mathrm{e}^{-09 * * *}$ & 0,14 & $0,000992^{* * *}$ & $0,00182^{* *}$ \\
\hline 2 & $1,69 \mathrm{e}^{-09 * * *}$ & $0,00774^{* *}$ & $0,00111^{* *}$ & $0,000382^{* * *}$ \\
\hline 3 & $1,56 \mathrm{e}^{-10 * * *}$ & $0,00839^{* *}$ & $1,48 \mathrm{e}^{-06 * * *}$ & $0,00219^{* *}$ \\
\hline 4 & $5,58 \mathrm{e}^{-09 * * *}$ & $0,00502^{* *}$ & $4,11 \mathrm{e}^{-06 * * *}$ & 0,17 \\
\hline 6 & $1,83 \mathrm{e}^{-06 * * *}$ & 0,124 & $0,000692^{* * *}$ & 0,413 \\
\hline 8 & $7,79 \mathrm{e}^{-08 * * *}$ & $0,0438^{*}$ & $0,000493^{* * *}$ & $0,0181^{*}$ \\
\hline
\end{tabular}

Примечание: статистическая значимость выявленных зависимостей: ${ }^{*} p \leq 0,05 ;{ }^{* *} p \leq 0,01 ;{ }^{* * *} p \leq 0,001$. 


\section{ЛИТЕРАТУРА}

1. Occupational noise exposure and hearing: a systematic review / A. Lie, M. Skogstad, H.A. Johannessen [et al.] // Int. Arch. Occup. Environ. Health. - 2016. - Vol. 89. P.351-372.

2. Аденинская, E.E. Научное обоснование и разработка модели медицинского наблюдения за работниками, занятыми в условиях воздействия шума: дис. ... канд. мед. наук: 14.02.04 / Аденинская Елена Евгеньевна. Москва, 2013. - 216 с.

3. Modelling the risk of noise-induced hearing loss among military pilots / P. Kuronen, E. Toppila, J. Starck [et al.] // Int. J. Audiol. - 2004. - Vol. 43. - P.79-84.

4. Hearing deficit in a birth cohort of U.S. male commuter air carrier and air taxi pilots / Y. Qiang, G.W. Rebok, S.P. Baker, G. Li // Aviat. Space Environ. Med. - 2008. Vol. 79. - P.1051-1055.

5. Hearing loss induced by occupational and impulse noise: results on threshold shifts by frequencies, age and gender from the Nord-Trondelag Hearing Loss Study / K. Tambs, H.J. Hoffman, H.M. Borchgrevink [et al.] // Int. J. Audiol. 2006. - Vol. 45. - P.309-317.

6. Федеральные клинические рекомендации по диагностике, лечению и профилактике потери слуха, вызванной шумом / И.В. Бухтияров, А.Ю. Бушманов, Е.Е. Аденинская [и др.] // Медицина труда и промышленная экология. - 2016. - № 3. - С.37-48.

7. Аденинская, Е.Е. Проблемы диагностики и факторы формирования нарушений слуха у членов летных экипажей гражданской авиации: дис. ... д-ра мед. наук: 14.01.03 / Аденинская Елена Евгеньевна. - Москва, 2018. - 330 c.

\section{REFERENCES}

1. Lie A, Skogstad M, Johannessen HA, et al. Occupational noise exposure and hearing: a systematic review. Int Arch Occup Environ Health. 2016; 89: 351-372.
2. Adeninskaya YeYe. Nauchnoye obosnovaniye i razrabotka modeli meditsinskogo nablyudeniya za rabotnikami, zanyatymi $v$ usloviyakh vozdeystviya shuma [Scientific substantiation and development of a model for medical observation of workers employed in conditions of exposure to noise]. Moskva: Nauchno-issledovatel'skiy institut meditsiny truda RAMN [Moscow: State Institution Research Institute of Occupational Medicine RAMS]. 2013; 216 p.

3. Kuronen P, Toppila E, Starck J, et al. Modelling the risk of noise-induced hearing loss among military pilots. Int $\mathrm{J}$ Audiol. 2004; 43: 79-84.

4. Qiang Y, Rebok GW, Baker SP, Li G. Hearing deficit in a birth cohort of US male commuter air carrier and air taxi pilots. Aviat Space Environ Med. 2008; 79: 1051-1055.

5. Tambs K, Hoffman HJ, Borchgrevink HM, et al. Hearing loss induced by occupational and impulse noise: results on threshold shifts by frequencies, age and gender from the Nord-Trøndelag Hearing Loss Study. Int J Audiol. 2006; 45: 309-317.

6. Buhtijarov IV, Bushmanov AJu, Adeninskaya EE. Federal'nye klinicheskie rekomendacii po diagnostike, lecheniju i profilaktike poteri sluha, vyzvannoj shumom [Federal clinical guidelines for the diagnosis, treatment and prevention of hearing loss caused by noise]. Medicina truda i promyshlennaja jekologija [Occupational medicine and industrial ecology]. 2016; 3: 37-48.

7. Adeninskaya YeYe. Problemy diagnostiki i faktory formirovaniya narusheniy slukha u chlenov letnykh ekipazhey grazhdanskoy aviatsii [Diagnostic problems and the formation factors of hearing impairment in members of flight crews of civil aviation]. Moskva: Nauchnoklinicheskiy tsentr otorinolaringologii Federal'nogo mediko-biologicheskogo agentstva [Moscow: Federal State Budgetary Institution Scientific-Clinical Center for Otorhinolaryngology of the Federal Medical and Biological Agency]. 2018; 330 p.

\title{
ОЦЕНКА УРОВНЕЙ АНТИМИКРОБНЫХ ПЕПТИДОВ В КРОВИ У ЖЕНЩИН РЕПРОДУКТИВНОГО ВОЗРАСТА С ВОСПАЛИТЕЛЬНЫМИ ЗАБОЛЕВАНИЯМИ ОРГАНОВ МАЛОГО ТАЗА В ЗАВИСИМОСТИ ОТ СОСТАВА МИКРОФЛОРЫ УРОГЕНИТАЛЬНОГО ТРАКТА
}

БОШЯН РОБЕРTA ОВИкОВНА, ORCID ID: 0000-0001-7707-0005; врач-акушер-гинеколог ООО «Центр клинической фармакологии и фармакотерапии», аспирант кафедры клинической фармакологии с курсом ДПО ФГБОУ ВО “Ставропольский государственный медицинский университет» Минздрава России, Россия, 355020, Ставрополь, ул. Мира, 310, тел. 8(962)459-80-90, e-mail: e-mail: ms.roberta@inbox.ru БАТУРИН ВЛАДИМИР АЛЕКСАНДРОВИЧ, ОRCID ID: 0000-0001-6013-6944; докт. мед. наук, профессор, зав. кафедрой клинической фармакологии с курсом ДПО ФГБОУ ВО «Ставропольский государственный медицинский университет» Минздрава России, Россия, 355020, Ставрополь, ул. Мира, 310 , e-mail: prof.baturin@gmail.com

\begin{abstract}
Реферат. Цель исследования - изучение уровней а-десензина-1 и ß-дефензина-1 в крови у пациенток репродуктивного возраста при воспалительных заболеваниях органов малого таза в зависимости от состава патогенной микрофрлоры урогенитального тракта. Материал и методы. В исследовании приняли участие 120 женщин репродуктивного возраста с воспалительными заболеваниями органов малого таза. Проводили количественное определение а-дефензина-1 (DEFa1) и ß-дефензина-1 (DEFb1) в сыворотке крови больных с использованием наборов для иммуноферментного анализа (Cloud-Clone Corporation, США). Критерии исключения: гонорейный цервицит, герпетический цервицит. Результаты и их обсуждение. У пациенток с изолированно выявленными возбудителями (Enterococcus faecalis, Candida spp., Ureaplasma spp., Chlamydia trachomatis Gardnerella vaginalis, Enterobacteriaceae) содержание а-десензина-1 в крови было относительно низким, хотя превышающим нормальные значения (R. Craddock, J. Huang, E. Jackson), а уровень $\beta$-дефензина-1 был высоким. В свою очередь, у женщин с микрофлорой, в которой превалировали микробные ассоциации, концентрация а-дефензина-1 была более высокой, а уровень $\beta$-дефензина-1 - низким по сравнению с нормальными значениями (J. Schneider,
\end{abstract}

